\title{
Plastic flow and failure in single point incremental forming of PVC sheets
}

\author{
S. Alkas Yonan ${ }^{1}$, M. B. Silva' ${ }^{2}$ P. A. F. Martins ${ }^{2 *}$, A. E. Tekkaya ${ }^{1}$ \\ ${ }^{1}$ Institute of Forming Technology and Lightweight Construction, Technical University of Dortmund, Baroper Str. 301, \\ D-44227 Dortmund, Germany \\ ${ }^{2}$ Instituto Superior Técnico, Universidade de Lisboa, Av. Rovisco Pais, 1049-001 Lisboa, Portugal
}

Received 20 October 2013; accepted in revised form 16 December 2013

\begin{abstract}
This paper presents an innovative and effective methodology to characterize plastic flow and failure in single point incremental forming (SPIF) of polymers that allows determining the stresses and the accumulated values of ductile damage directly from the experimental values of strain at various positions over the deformed polymer sheets. The approach traces the deformation path of material elements in conical and pyramidal SPIF parts, undergoing linear strain loading paths from beginning until failure, and is built upon the generalization of the analytical framework conditions assumed by Glover et al. [1] to the pressure-sensitive yield surfaces of polymers under incompressible, non-associated, plastic flow. Experimentation in conventional and multi-stage SPIF of Polyvinylchloride (PVC) sheets confirms the effectiveness of the proposed methodology and demonstrates that standard non-coupled damage models currently utilized in sheet metal forming are inapplicable to describe failure in polymers. Instead fracture forming limit lines (FFL's) should be employed.
\end{abstract}

Keywords: processing technologies, incremental sheet forming, polyvinylchloride (PVC), damage, fracture

\section{Introduction}

Current manufacturing technologies for producing polymer parts are based on heating-shaping-cooling process routes and are closely linked to mass production. The first attempt to develop a flexible, costefficient technology to allow small batch production of polymer parts was performed by Franzen et al. [2], who evaluated the performance of single point incremental forming (SPIF) to shape commercial Polyvinylchloride (PVC) sheets at room temperature (Figure 1a). Table 1 presents a comparative summary of the main technical and economic aspects of different polymer processing technologies.

Franzen et al. [2] identified the main operating parameters of the process and systematized three different modes of failure, giving special emphasis to the development of cracks in conditions that are different from those commonly found in the SPIF of metals.

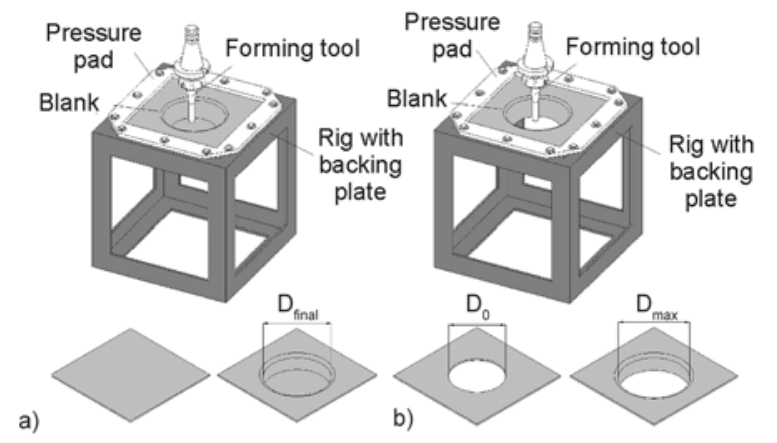

Figure 1. Schematic representation of the experimental apparatus utilized in single point incremental forming (SPIF) for producing (a) a conical part and (b) a hole-flanged cylindrical part in a blank with a pre-cut hole

\footnotetext{
${ }^{*}$ Corresponding author, e-mail: pmartins@ist.utl.pt

(C) BME-PT
} 
Table 1. Technic and economic aspects of polymer processing

\begin{tabular}{|l|l|l|l|c|l|}
\hline & $\begin{array}{c}\text { Equipment } \\
\text { cost }\end{array}$ & $\begin{array}{c}\text { Tooling } \\
\text { cost }\end{array}$ & $\begin{array}{c}\text { Production } \\
\text { rate }\end{array}$ & $\begin{array}{c}\text { Batch size } \\
\text { (number of parts) }\end{array}$ & \multicolumn{1}{|c|}{ Applications } \\
\hline Blow moulding & Medium & Medium & Medium & $>10^{3}$ & $\begin{array}{l}\text { Hollow thin-walled parts such as bottles, con- } \\
\text { tainers and fuel tanks }\end{array}$ \\
\hline Compression moulding & High & Medium & Medium & $>10^{3}$ & $\begin{array}{l}\text { Parts similar to closed-die forgings primarily } \\
\text { made from highly filled polymers with a high } \\
\text { viscosity }\end{array}$ \\
\hline Injection moulding & High & High & High & $>10^{4}$ & $\begin{array}{l}\text { Complex shapes of various sizes with fine } \\
\text { details such as gears, fasteners, housings, } \\
\text { switches and knobs }\end{array}$ \\
\hline Transfer moulding & High & High & Medium & $>10^{3}$ & $\begin{array}{l}\text { More complex parts than those produced by } \\
\text { compression moulding }\end{array}$ \\
\hline Thermoforming & Low & Low & Low & $>10^{2}$ & $\begin{array}{l}\text { Parts with shallow or deep cavities made from } \\
\text { polymer sheets such as packaging cases }\end{array}$ \\
\hline SPIF & $(*)$ & Very Low & Low & $>1$ & $\begin{array}{l}\text { Rapid prototypes and low batch production of } \\
\text { simple polymer sheet parts similar to those fab- } \\
\text { ricated by thermoforming }\end{array}$ \\
\hline
\end{tabular}

$(*)$ Does not require a specific piece of equipment because $\mathrm{CNC}$ machining centers are commonly available at industrial companies.

In a subsequent publication, Le et al. [3] presented a set of experimental results in SPIF of Polypropylene (PP) sheets and utilized statistical analysis based on design of experiments to study the influence of the step size, tool size, feed rate and spindle speed on the overall formability of the process. Two modes of failure similar to those that had been previously observed by Franzen et al. [2] were reported. In a second paper, Martins et al. [4] extended the scope of their investigation to include four additional polymers (polyoxymethylene (POM), polyethylene (PE), polyamide (PA) and polycarbonate (PC)). The previously identified failure modes were revisited in the light of new experimental data and a criterion for the selection of polymers for SPIF applications was proposed.

Taken together at the end of 2009, the results of the abovementioned preliminary studies allowed anticipating that research in the SPIF of polymers would continue to be carried out in two different approaches. Firstly, to evaluate the performance and potential applications of different polymers under new and increasingly sophisticated sets of operating parameters. Secondly, to develop a new fundamental level of understanding about the SPIF of polymers by means of analytical or numerical models that could be able to predict plastic flow and failure across the useful range of operating conditions.

The work of Silva et al. [5] in hole-flanging of polyethylene terephthalate (PET) and (PC) sheets by means of multistage SPIF is a good example of the first line approach and contributed to enlarging the scope of applications of the SPIF of polymers to the production of hole-flanged parts with vertical walls (Figure 1b). The discussion on the relative performance of the SPIF of polymers against that of metals performed by Silva et al. [6] may also be included in the first line approach (Table 2).

The works of Silva et al. [7] and Alkas Yonan and coworkers $[8,9]$ were built upon the second line approach. Silva et al. [7] developed a theoretical framework based on membrane analysis and pressure-sensitive yield surfaces to characterize plastic flow and failure in polymer sheets. Alkas Yonan and coworkers $[8,9]$ developed a non-linear viscoplastic material model for thermoplastics and its finite strain extension to the finite element simulation of PVC parts produced by SPIF.

However, both developments exhibited difficulties in modelling the SPIF of polymers. For example, the theoretical framework proposed by Silva et al. [7] could only be qualitatively assessed against experimental data and observations. On the other hand,

Table 2. Experimental maximum drawing angles $\psi_{\max }$ that metal and polymer sheet blanks can undertake when producing a truncated conical shape by SPIF

\begin{tabular}{|c|c|c|}
\hline \multicolumn{2}{|c|}{ Material } & \multirow{2}{*}{$\begin{array}{c}\begin{array}{c}\psi_{\max } \\
{\left[^{\circ}\right]}\end{array} \\
80^{\circ}\end{array}$} \\
\hline \multirow{3}{*}{ Aluminium } & AA1050-O & \\
\hline & AA1050-H111 & $76^{\circ}$ \\
\hline & AA5052 & $74^{\circ}$ \\
\hline \multirow{3}{*}{ Steel } & DC01 & $76^{\circ}$ \\
\hline & DC04 & $74^{\circ}$ \\
\hline & AISI 304L & $74^{\circ}$ \\
\hline Titanium & Grade 2 & $65^{\circ}$ \\
\hline \multirow{2}{*}{ Polymer } & PET & $\sim 90^{\circ}$ \\
\hline & PVC & $66^{\circ}$ \\
\hline
\end{tabular}


the evaluation of the non-linear viscoplastic material model proposed by Alkas Yonan and coworkers $[8,9]$ was limited by small deformations, which prevented analysing plastic flow and the corresponding stress field for high values of strain.

As a result of the abovementioned limitations, the aims and scope of this paper is to present an alternative and effective methodology to characterize plastic flow and failure in the SPIF of polymers. The proposed methodology traces the deformation path of material elements in conical and pyramidal benchmark parts produced by SPIF and is built upon the generalization of the analytical framework proposed by Glover et al. [1], in their investigation on failure maps in sheet metal forming, to pressuresensitive yield surfaces under non-associated plastic flow.

In general, the proposed methodology to be presented in this paper will provide quantitative answers to important key questions related to the SPIF of polymers that were never solved or properly addressed before; what is the stress distribution and how can these values be determined from the experimental values of strain at various positions over the polymer SPIF parts? How can the critical values of ductile damage based on standard non-coupled models be experimentally determined without having the need to apply inverse calibration procedures? Is there a correlation between the aforementioned values of ductile damage at the onset of necking and the fracture forming line (FFL)? Can the FFL be successfully utilized for assessing the formability limits of multi-stage SPIF of polymer sheets? The organization of the paper is the following. Section 2. summarises the experimental procedures utilized in the mechanical and formability characterization of the material, presents the fundamentals of the new proposed methodology to determine the distributions of stress and ductile damage at the onset of cracking, and describes the work plan utilized in the experiments that give support to the paper. Section 3. presents and discusses the results obtained in SPIF of cones and pyramids, analysing the strain loading paths in the principal strain space in the light of non-coupled ductile damage criteria and the FFL. The effectiveness of the FFL is subsequently evaluated by means of experiments in holeflanging produced by multi-stage SPIF. Section 4 . presents the conclusions.

\section{Experimental}

\subsection{Determination of the stress-strain curve}

The research work was performed on sheets of polyvinylchloride (PVC) with $3 \mathrm{~mm}$ thickness (commercial designation Koemadur WA 155, supplier Koemmerling, Germany). The choice of PVC results from its high strength, good dimensional stability and more environmental friendly features than alternative polymers due to its lower dependency on petroleum (only $40 \%$ of the composition of PVC is petroleum-derived).

The stress-strain curves of PVC were obtained by means of tensile and stack compression tests. The specimens utilized in the tensile tests were machined from the supplied sheets in accordance to the ISO 527 standard. The testing speed was equal to $0.2 \mathrm{~mm} / \mathrm{min}$. The multi-layer cylinder specimens utilized in the stack compression tests were assembled by piling up 3 circular discs with $12 \mathrm{~mm}$ diameter and $3 \mathrm{~mm}$ thickness cut from the aforementioned PVC sheets by water jet. The testing speed was equal to $0.02 \mathrm{~mm} / \mathrm{min}$.

Figure 2 shows the tensile and compressive stressstrain curves of PVC. It is worth noting that the engineering stress-strain curves resulting from the tensile and stack compression tests were converted into true stress-strain curves in order to be employed in the analytical methodology to characterize plastic flow and failure that will be described in section 2.2. The relationship between true stress and true strains for both tensile and stack compression tests were approximated by a Ludwik-Hollomon's model $\sigma=K \varepsilon^{\text {n }}$ (Figure 2) assuming PVC as incom-

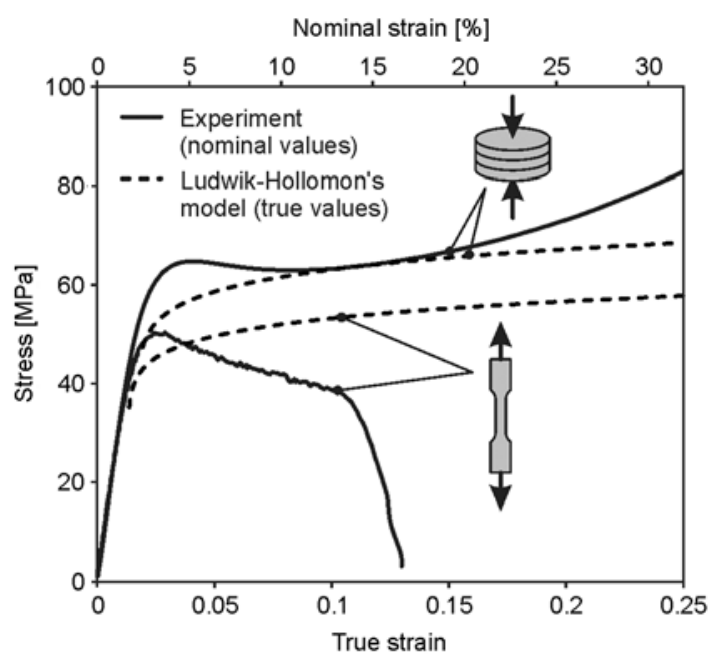

Figure 2. Stress-strain curves of PVC obtained from tensile and stack compression tests 
Table 3. Representative summary of the mechanical properties of the PVC sheets that were utilized in the investigation

\begin{tabular}{|l|c|c|c|c|}
\hline & $\begin{array}{c}\text { Elasticity modulus } \\
\text { (tension), } \\
{[\mathbf{M P a}]}\end{array}$ & $\begin{array}{c}\text { Elasticity modulus } \\
\text { (compression), } \mathbf{E} \\
{[\mathbf{M P a}]}\end{array}$ & $\begin{array}{c}\text { Yield stress (tension), } \boldsymbol{\sigma}_{\mathbf{Y T}} \\
{[\mathbf{M P a}]}\end{array}$ & $\begin{array}{c}\text { Yield stress } \\
\text { (compression), } \\
\text { [MPa] }\end{array}$ \\
\hline Average value & 2760.0 & 2712.27 & 38.63 & 46.67 \\
\hline Standard deviation & 58.3 & 10.90 & 1.05 & 3.06 \\
\hline
\end{tabular}

pressible (see Equation (1)). This was necessary in order to facilitate mathematical treatment by means of the proposed methodology to be described in section 2.2.:

Tensile test:

$$
\begin{aligned}
& \sigma=65 \varepsilon^{0.082}[\mathrm{MPa}] \\
& \sigma=77 \varepsilon^{0.08}[\mathrm{MPa}]
\end{aligned}
$$

The difference between the tensile and compressive stress-strain curves is attributed to the strength differential effect that is caused by the pressure-sensitive characteristics of the yield surface. Table 3 presents a summary of the mechanical properties of PVC.

\subsection{Determination of strains, stresses and ductile damage}

The experimental technique utilized for determining the in-plane strains on the surface of the specimens produced by SPIF made use of imprinted grids of dots with $2 \mathrm{~mm}$ initial spacing between dot centres (Figure 3). During SPIF, the grid deforms with the material, and the corresponding major and minor surface strains were automatically measured from the deformed pattern by employing a digital image correlation technique based on a commercial Argus system of GOM mbH that compares the actual grid with the original (undeformed) grid.

In general, sheet metal forming is characterized by complex strain paths that prevent the distribution of stresses and the critical values of damage at the onset of necking to be directly determined from the experimental values of strain. However, in case of
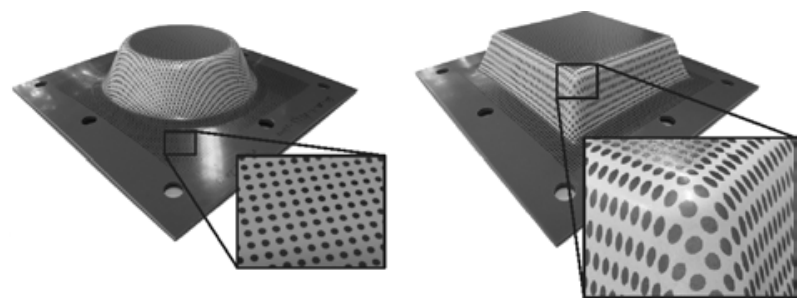

Figure 3. Conical and pyramidal parts produced by SPIF with a constant drawing angle $\psi=60^{\circ}$ showing details of the undeformed and deformed grid of dots that were imprinted on the surface of the blanks before testing conical and pyramidal SPIF parts made from PVC, previous work in the field combined with results obtained in the present investigation showed that strain paths are linear and do not experience abrupt changes in direction at the forming limit curve (FLC) [7].

The aforementioned observation opens the possibility of utilizing SPIF tests based on conical and pyramidal geometries to set up a new methodology for determining the stress distribution and the critical values of damage from the experimental strains at the onset of cracking. In specific terms the proposed approach makes use of the pressure modified version of the von Mises yield criterion (see Equation (2)) that explicitly accounts for the stress differential effect of polymers [10]:

$F\left(\sigma_{\mathrm{ij}}\right)=\bar{\sigma}^{2}-\sigma_{\mathrm{C}} \cdot \sigma_{\mathrm{T}}+\left(\sigma_{\mathrm{C}}-\sigma_{\mathrm{T}}\right) \sigma_{\mathrm{kk}}=0$

where $F\left(\sigma_{\mathrm{ij}}\right)$ is the yield function, $\bar{\sigma}=\sqrt{\frac{1}{2} \sigma_{\mathrm{ij}}^{\prime} \sigma_{\mathrm{ij}}^{\prime}}$ is the effective stress, $\sigma_{\mathrm{ij}}^{\prime}$ is the deviatoric stress and $\sigma_{\mathrm{kk}}=\delta_{\mathrm{ij}} \sigma_{\mathrm{ij}}$. The tensile $\sigma_{\mathrm{T}}$ and compressive $\sigma_{\mathrm{C}}$ flow stresses are obtained from tension and compression tests (Section 2.1.) and in case $\sigma_{\mathrm{T}}=\sigma_{\mathrm{C}}$, the traditional von Mises criterion (currently utilized in metals) results.

Because PVC is nearly incompressible [11], the normality rule typical of associated plasticity does not hold in case of pressure dependent yield surfaces (Equation (2)) since it leads to volume variations that are at least one order of magnitude larger than observed experimental values [12]. Instead, a non-associated flow rule where the plastic potential is taken as $J_{2}$ of the von-Mises plasticity should be employed $[11,13]$.

Under these circumstances, and assuming isotropic plastic deformation under plane stress conditions $\sigma_{3}=0$, the constitutive equations relating the inplane strain increments with the applied stresses are given by Equation (3):

$\mathrm{d} \varepsilon_{1}=\frac{\mathrm{d} \bar{\varepsilon}}{\bar{\sigma}}\left(\sigma_{1}-\frac{1}{2} \sigma_{2}\right), \mathrm{d} \varepsilon_{2}=\frac{\mathrm{d} \bar{\varepsilon}}{\bar{\sigma}}\left(\sigma_{2}-\frac{1}{2} \sigma_{1}\right)$ 
where the effective stress $\bar{\sigma}$ and the effective strain increment $\mathrm{d} \bar{\varepsilon}$ are obtained from Equation (4):

$$
\begin{aligned}
\bar{\sigma} & =\sqrt{\sigma_{1}^{2}+\sigma_{2}^{2}-\sigma_{1} \sigma_{2}} \\
\mathrm{~d} \bar{\varepsilon} & =\frac{2}{\sqrt{3}} \sqrt{\mathrm{d} \varepsilon_{1}^{2}+\mathrm{d} \varepsilon_{2}^{2}+\mathrm{d} \varepsilon_{1} \mathrm{~d} \varepsilon_{2}}
\end{aligned}
$$

Now, taking into consideration that plastic deformation of PVC sheets in conical and pyramidal SPIF parts is carried out under linear strain-paths with a slope $\beta=\mathrm{d} \varepsilon_{2} / \mathrm{d} \varepsilon_{1}=\varepsilon_{2} / \varepsilon_{1}$, the following relationship can be derived as shown by Equation (5):

$\alpha=\frac{\sigma_{2}}{\sigma_{1}}=\frac{2 \beta+1}{2+\beta}$

and the simplified expressions of the principal inplane stresses $\left(\sigma_{1}, \sigma_{2}\right)$ can be obtained by replacing Equation (5) into Equation (4):

$\sigma_{1}=\frac{\bar{\sigma}}{\sqrt{\left(1-\alpha+\alpha^{2}\right)}}, \sigma_{2}=\frac{\alpha \bar{\sigma}}{\sqrt{\left(1-\alpha+\alpha^{2}\right)}}(6)$

The effective stress $\bar{\sigma}$ in Equation (6) is calculated by combining the tensile and compressive flow stresses from Equation (1) into Equation (2) with the integration of the increment of effective strain $\bar{\varepsilon}=\int \mathrm{d} \bar{\varepsilon}$ up to the actual plastic deformation.

Once the distribution of stress is determined from the experimental measurements of the major inplane strains, the critical values of damage $D^{\text {crit }}$ at the onset of failure are easily obtained by integrating the damage function $D$ from beginning until failure (that is, until the amount of deformation that corresponds to the instant of time where cracking occurs).

The present work makes use of the normalized Cockcroft-Latham [14] ductile fracture criterion and of two other ductile fracture criteria that are based on the triaxiality ratio $\sigma_{\mathrm{m}} / \bar{\sigma}$ that was originally proposed by McClintock [15] and is known to play an important role in the overall formability. The damage functions $D_{\mathrm{CL}}, D_{\mathrm{RT}}$ and $D_{\mathrm{A}}$ of the ductile fracture models proposed by Cockcroft and Latham [14], Rice and Tracey [16] and Ayada et al. [17], take the form given by Equation (7):

$$
\begin{aligned}
& D_{\mathrm{CL}}=\int_{0}^{\bar{\varepsilon}_{\mathrm{f}}} \frac{\sigma_{1}}{\bar{\sigma}} \mathrm{d} \bar{\varepsilon}, D_{\mathrm{RT}}=\int_{0}^{\bar{\varepsilon}_{\mathrm{f}}} \exp \left(\frac{3}{2} \frac{\sigma_{\mathrm{m}}}{\bar{\sigma}}\right) \mathrm{d} \bar{\varepsilon} \\
& D_{\mathrm{A}}=\int_{0}^{\bar{\varepsilon}_{\mathrm{f}}} \frac{\sigma_{\mathrm{m}}}{\bar{\sigma}} \mathrm{d} \bar{\varepsilon}
\end{aligned}
$$

where $\sigma_{\mathrm{m}}=\sigma_{\mathrm{kk}} / 3$ is the average stress and $\bar{\varepsilon}_{\mathrm{f}}$ is the effective strain at fracture.

The overall methodology could have been extended to other ductile fracture criteria available in the literature but its application would basically remain identical.

\subsection{Determination of the fracture forming limit line}

The formability limits at fracture (fracture forming limit line, FFL) were determined by combining the results obtained from independent tensile and hydraulic bulge tests with the experimental measurements performed on conical and pyramidal SPIF parts at the onset of cracking.

The tensile tests were performed in an Instron 4507 testing machine and the hydraulic bulge tests were performed in a universal sheet testing machine (Erichsen 145/60) using a bi-axial circular (100 mm) tooling set. The conical and pyramidal SPIF parts were produced with a forming tool with a hemispherical tip of $10 \mathrm{~mm}$ diameter. The tests were performed with helical tool paths characterized by a step size per revolution equal to $0.5 \mathrm{~mm}$ (downward feed) and a feed rate equal to $1000 \mathrm{~mm} / \mathrm{min}$. The rotation of the forming tool was free and the lubricant was deep-drawing oil.

The experimental strains at the onset of fracture resulting from the above mentioned procedure were fitted by a straight line (the fracture forming limit line, FFL) falling from left to right and bounded by grey areas corresponding to an interval of approximately $10 \%$ (above and below the FFL) due to the experimental uncertainty in its determination (see Figure 4 and Equation (8)):

$\varepsilon_{1}=-0.19 \varepsilon_{2}+0.87$

The aforementioned uncertainty stems from the utilization of different tests to the determination of the FFL and from difficulties in measuring the strain gauge strains at the onset of fracture in case of tensile and hydraulic bulge tests.

As shown in Figure 4, the FFL of PVC presents a slope considerably different from that commonly observed in metals, which meet the dual condition of constant thickness strain and constant triaxiality ratio $\sigma_{\mathrm{m}} / \bar{\sigma}$ at fracture (corresponding to a slope equal to ' -1 ', [18]). In fact, the experimental slope equal to ' -0.19 ' corresponds to a diminishing rate 


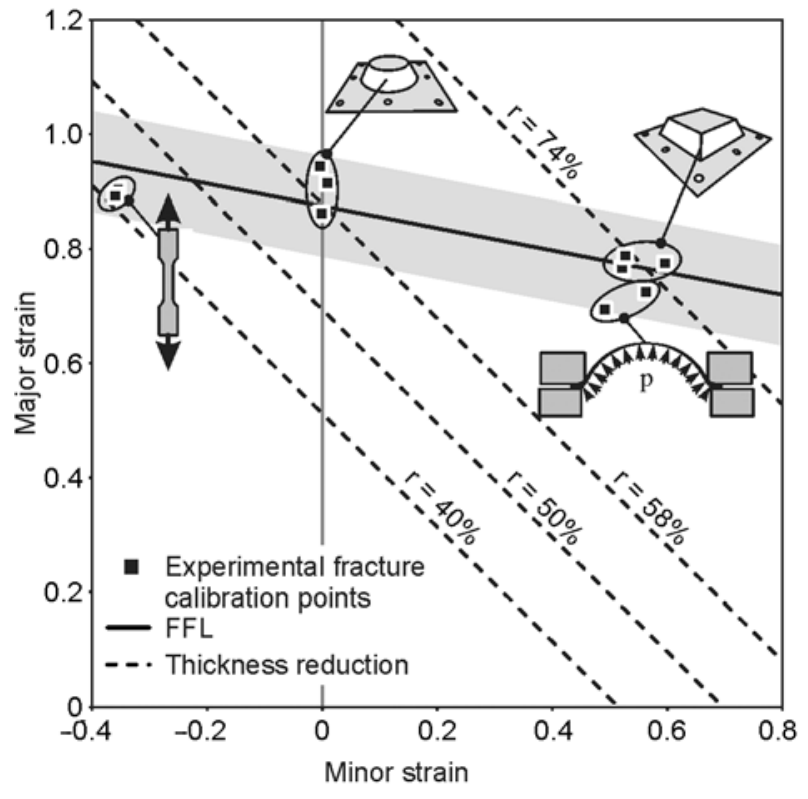

Figure 4. Fracture forming limit line (FFL) and iso-thickness reduction lines of the PVC sheets (in \%)

of the accumulated damage when the strain loading paths evolve from the tension-compression (second quadrant) to the tension-tension (first quadrant) regions of the principal strain space.

The dashed grey lines in Figure 4 correspond to the iso-thickness reduction lines and are calculated from the incompressibility condition of the PVC sheets shown in Equation (9):

$\varepsilon_{1}+\varepsilon_{2}=-\ln \left(1-\frac{r}{100}\right)$

where $r=\left(t_{0}-t\right) / t_{0}$ is the reduction in sheet thickness (in \%).
As seen in Figure 4, the reduction in sheet thickness at the onset of failure by fracture changes from $58 \%$ to $74 \%$ when the strain loading paths change from plane strain to biaxial stretching. This result is different from what is commonly observed in metals and, as shown in Section 3., will have a major influence on the correlation between the FFL and the standard non-coupled ductile damage criteria due to Cockcroft and Latham [14], Rice and Tracey [16] and Ayada et al. [17].

\subsection{Fabrication of hole-flanged parts by multi-stage SPIF}

The experiments in hole-flanging produced by multi-stage SPIF made use of rectangular PVC blanks with $220 \mathrm{~mm}$ side length and $3 \mathrm{~mm}$ thickness. All the blanks were cut from the supplied sheets and subsequently drilled to provide holes at the centre with diameters of 80 and $90 \mathrm{~mm}$. Drilling was performed with a hole cutter and the resulting edges were carefully grinded with medium and fine grit sand paper to remove any sharp edges or burrs. The single point forming tool and the basic operating conditions utilized in the experiments were similar to those previously utilized for fabricating the conical and pyramidal SPIF parts (Section 2.3.).

The experimental work plan was designed with a twofold objective. Firstly, to investigate the feasibility of using multi-stage SPIF to produce holeflanges in PVC blanks with pre-cut holes. Secondly, to analyse the feasibility of using the FFL to assess the formability of PVC under complex strain paths

Table 4. Experimental work plan for producing hole-flanged PVC parts by multi-stage SPIF

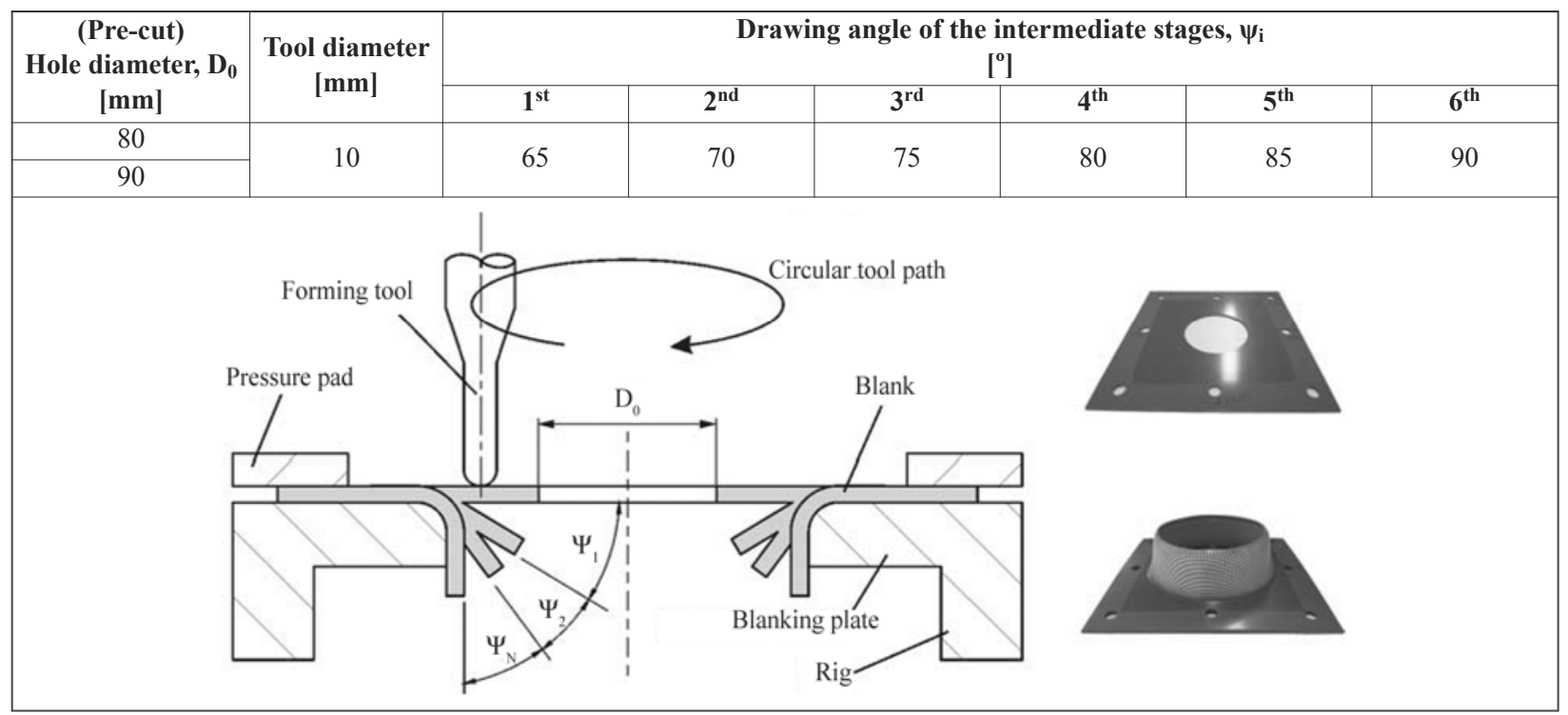


resulting from multi-stage SPIF. Each individual forming stage was produced with a constant drawing angle that was increased by $\Delta \psi=5^{\circ}$, between stages, from an initial value $\psi_{1}=65^{\circ}$ until a limiting value $\psi_{\mathrm{n}}=90^{\circ}$, corresponding to a hole-flange with vertical walls. Table 4 presents a summary of the experimental work plan.

\section{Results and discussion}

\subsection{Plastic flow and failure}

The experimental values of the major and minor inplane true strains of selected conical and pyramidal parts produced by SPIF were measured along the meridional cross section in accordance to the method and procedure described in Section 2.2. after the parts reach failure or, in case of no-failure, at the end of the process. The results are plotted in the principal strain space (Figure 5).

As seen in Figure 5a the average values of the experimental in-plane true strains of the conical parts are aligned along the vertical axis corresponding to $\varepsilon_{2}=0$ and confirm that plastic flow takes
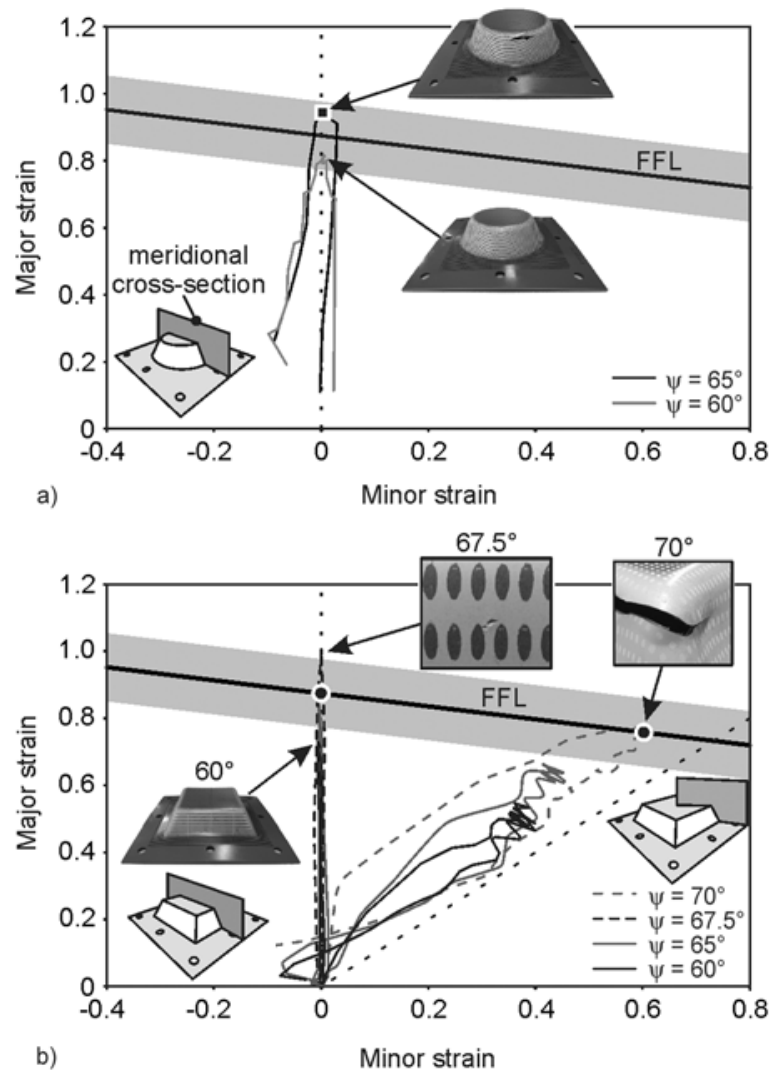

Figure 5. Experimental in-plane true strains in (a) conical and (b) pyramidal PVC parts produced by SPIF with different drawing angles. The photographs show a successful pyramidal part and details of the cracks that were found in two different regions place under plane strain proportional (or linear, $\beta=$ $\mathrm{d} \varepsilon_{2} / \mathrm{d} \varepsilon_{1}=0$ ) loading conditions.

The agreement between the maximum values of strain at the onset of cracking and the FFL that was previously determined in Section 2.3. is very good. In fact, the maximum in-plane true strains of the conical part produced by SPIF with a drawing angle $\psi=65^{\circ}$, which failed by cracking along the circumferential direction (refer to the photograph in Figure $5 \mathrm{a}$ ), are located above the FFL but inside the grey scattered area of $10 \%$ resulting from the aforementioned uncertainty in the determination of the FFL. In contrast, the maximum in-plane true strains of the other conical part produced by SPIF with a drawing angle $\psi=60^{\circ}$ are located below the FFL, as expected from a PVC part that was successfully fabricated by SPIF.

The results obtained for the pyramidal parts produced by SPIF further confirm the good agreement between the experimental values of the in-plane true strains at the onset of failure and the FFL. For example, the strain values of the pyramidal part produced by SPIF with a drawing angle $\psi=67,5^{\circ}$, which failed by cracking at the mid-side (undergoing plane strain loading conditions), are located above the FFL and those of the pyramidal part produced by SPIF with a drawing angle $\psi=70^{\circ}$, which failed by cracking at the corner (undergoing biaxial strain loading conditions), are very close to the FFL. The photographs included in Figure 5b show the details of cracks that were observed in both cases.

It is worth noting that all the other pyramidal parts produced by SPIF with smaller drawing angles than those mentioned above present maximum in-plane true strain values below the FFL. These results are in close agreement with the absence of cracks. See, for example, the pyramidal part that was successfully produced by SPIF with a drawing angle $\psi=$ $60^{\circ}$ in Figure $5 \mathrm{~b}$.

The stress field corresponding to the aforementioned experimental values of strain at different locations over the conical and pyramidal PVC parts can be determined by means of the previously described methodology (Section 2.3.). Taken the conical part produced by SPIF with a drawing angle $\psi=65^{\circ}$ for example, the major and minor in-plane stresses are directly calculated from Equation (6) using an iterative analytical procedure based on the experimental values of the in-plane strains (refer to 
the black square mark in Figure 5a) and the tensile and compressive stress-strain curves of Equation (1).

The iterative procedure is as follows. Firstly, it is necessary to obtain a rough approximation $\sigma_{1}^{0}, \sigma_{2}^{0}$, $\sigma_{\mathrm{m}}^{0}=\sigma_{\mathrm{kk}}^{0} / 3$ of the major, minor and average stresses by applying Equation (6) in conjunction with the tensile stress-strain curve of Equation (1), which is then utilized to obtain a new set of values for $\sigma_{1}^{1}, \sigma_{2}^{1}$, $\sigma_{\mathrm{m}}^{1}=\sigma_{\mathrm{kk}}^{1} / 3$ that already take into consideration the strength differential effect of the PVC yield surface. The overall procedure is applied until convergence, as it is shown in Figure 6.

As seen in Figure 6, the relative error arising from the utilization of the first approximation of the inplane stresses, which were solely based on the tensile stress-strain curve of PVC, is rather small (below 10\%). This means that the strength differential effect of PVC may be neglected from calculation of plastic flow and failure, as it was previously done by Alkas Yonan and coworkers [8,9], who only considered the tensile stress-strain curve during the development of their non-linear viscoplastic material model for thermoplastics.

Hence, by taking the abovementioned simplification into account, the critical values $D^{\text {crit }}$ of the noncoupled ductile damage models proposed by Cockcroft and Latham [14], Rice and Tracey [16] and Ayada et al. [17] can be easy and straightforwardly determined by means of Equations (7), without the need to apply inverse, cumbersome, calibration procedures based on numerical simulations. The experimental in-plane true strains utilized in these calculations were taken from the intersection of the FFL and the vertical axis $\left(\varepsilon_{2}=0\right)$, and from the intersec-

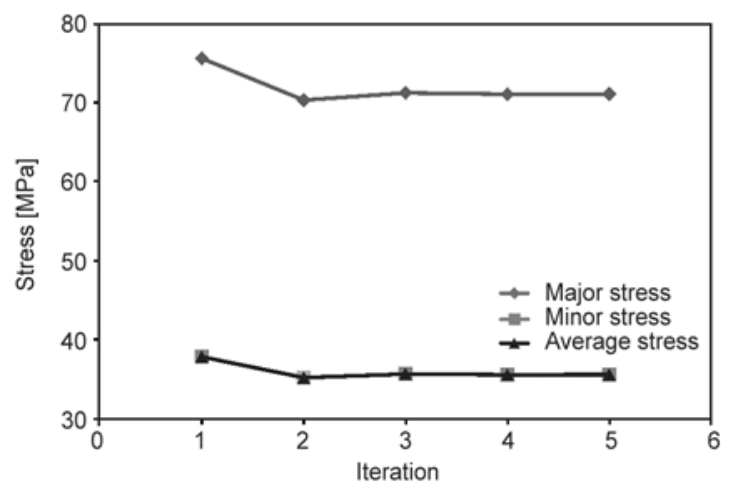

Figure 6. Iterative procedure for determining the major, minor and average stress values at the onset of cracking for a conical part produced by SPIF with a drawing angle $\psi=65^{\circ}$ tion of the FFL and the inclined strain-loading path corresponding to the corners of the pyramids $\left(\varepsilon_{1} \cong\right.$ $1.3 \varepsilon_{2}$ ) (refer to the black circular marks in Figures $5 b$ ). The results are shown in Figure 7.

As seen in Figure 7, and contrary to what has been recently observed in metals [19], the critical values of damage $D^{\text {crit }}$ are significantly influenced by the strain loading conditions (from $21 \%$ in case of the damage models proposed by Cockcroft and Latham to 55 and $53 \%$ in case of the other damage models proposed by Rice and Tracey and Ayada et al., respectively). The fact that the slope of the FFL in Equa-
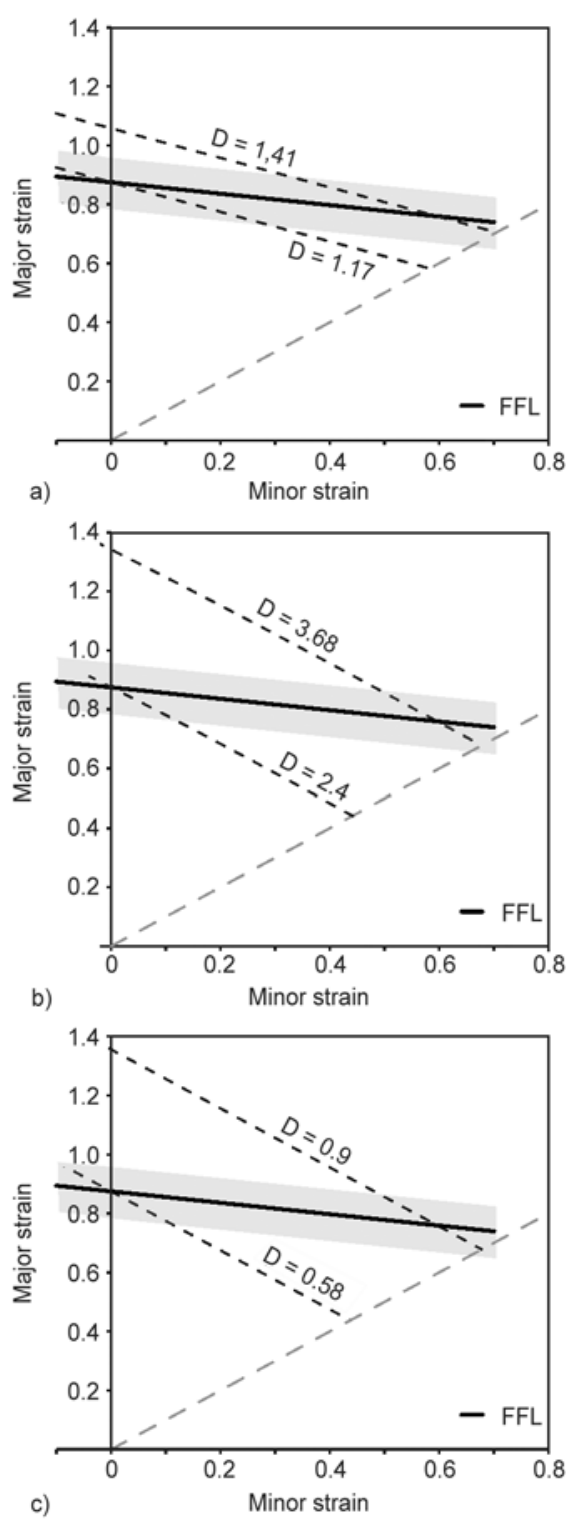

Figure 7. Principal strain space showing the FFL and two different black dashed lines corresponding to the iso-contours of the critical values of damage $D^{\text {crit }}$ according to (a) Cockcroft and Latham [14] (b) Rice and Tracey [16] and (c) Ayada et al. [17] criteria 


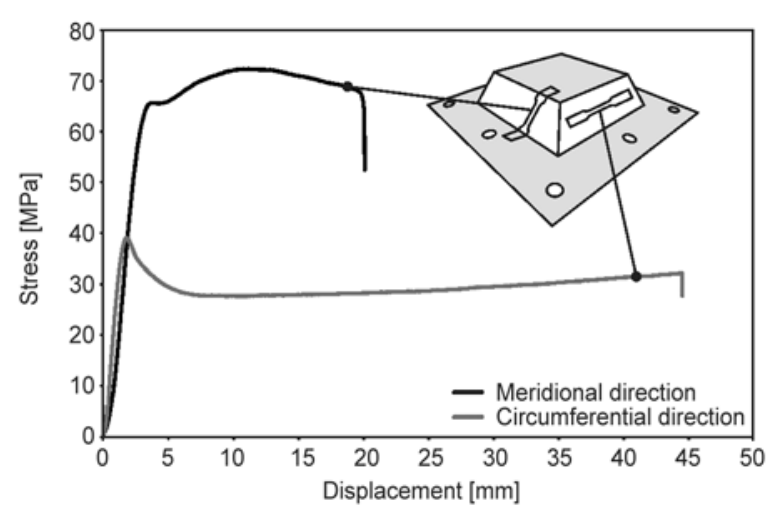

Figure 8. Stress-displacement curves obtained from tensile tests performed on specimens taken from the longitudinal and meridional directions along the mid-side of a pyramidal part produced by SPIF with a drawing angle $\psi=60^{\circ}$

tion (8) is significantly different from ' -1 ' provides a justification for the above mentioned conclusion because PVC does not follow the conditions of failure by cracking under constant thinning and constant triaxiality ratio $\sigma_{\mathrm{m}} / \bar{\sigma}$ that are typical of metals. The cause, however, is probably related to stable neck propagation during SPIF, a phenomenon that does not occur in metals.

Stable neck propagation along the meridional direction of PVC parts produced by SPIF is also responsible for inducing anisotropy that will result in different stress responses along the meridional and circumferential directions. This phenomenon is shown in Figure 8 and will have a major influence in multi-stage SPIF, as will be explained in the following section of the paper.

\subsection{Hole-flanging of PVC sheets produced by multi-stage SPIF}

The experiments in hole-flanging of PVC sheets produced by multi-stage SPIF were performed in accordance to the work plan and operating conditions that were previously described in Section 2.4. The black curves in Figures $9 a$ and $9 b$ are the envelopes of the greatest achievable experimental strains at selected forming stages in hole-flanging produced by SPIF. Each line corresponds to an intermediate drawing angle $\psi_{\mathrm{i}}$ listed in Table 2 and will be hereafter referred to as 'the strain envelope'. In case of the strain envelope corresponding to the last forming stage $\left(\psi_{1}=90^{\circ}\right)$ of Figure 9a, point ' $A$ ' corresponds to near plane strain conditions at the middle of the wall flange, point ' $B$ ' to biaxial strain conditions close to its edge and point ' $C$ ' to tensile
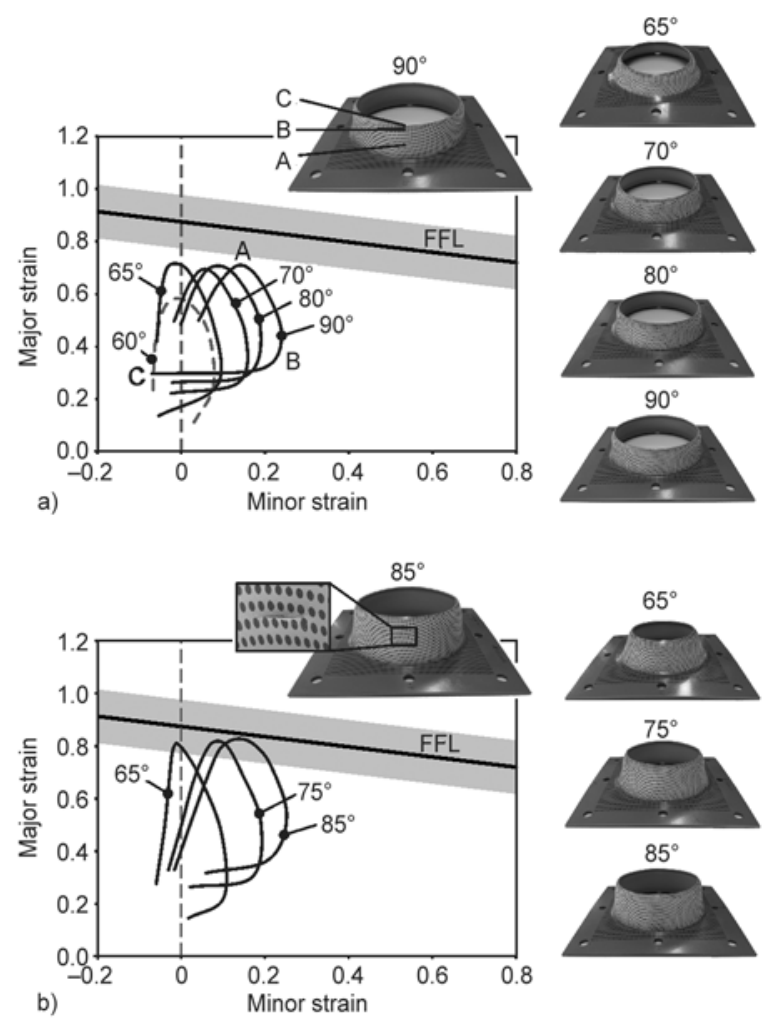

Figure 9. Experimental strain envelopes in the principal strain space for different intermediate configurations of hole-flanging produced by multi-stage SPIF. (a) Successful production using a PVC blank with an initial hole diameter of $90 \mathrm{~mm}$. (b) Failure by cracking at $\psi_{5}=85^{\circ}$ (refer to the detail) in case of a PVC blank with an initial hole diameter of $80 \mathrm{~mm}$.

strain conditions along a very narrow region located at the edge of the hole. This result is similar to what has recently been observed in the hole-flanging of metals [19].

A detailed analysis of the evolution of the strain envelopes from the initial drawing angle $\psi_{1}=65^{\circ}$ up to the last drawing angles included in Figures 9a and $9 \mathrm{~b}$, allows us to conclude that strain envelopes corresponding to intermediate forming stages with increasing values of $\psi_{\mathrm{i}}$ unveil significant differences between PVC and metals. In case of PVC, the second and following stages are characterized by expansion and translation of the strain envelopes towards higher values of the minor in-plane true strain $\varepsilon_{2}$. During expansion and translation the major in-plane true strain $\varepsilon_{1}$ remains approximately constant and equal to the values that had been reached during the first forming stage. This behaviour is different from that observed in metals, which experience expansion of the strain envelopes (from 
the origin) under proportional loading conditions $\beta=$ Const. [19].

As a result of this, the final height of the PVC wall flanges is practically identical to that reached at the end of the first forming stage. The subsequent forming stages mainly account for the increase in the diameter of the hole as a result of the decrease in sheet thickness $\left(\varepsilon_{2}=-\varepsilon_{3}\right)$, as the wall flange progressively rotates towards the vertical direction.

The cause of this behaviour is attributed to stable neck propagation during the first forming stage, which induces strong anisotropy and encourages plastic flow during the subsequent forming stages to be preferentially carried out in the circumferential direction instead of the meridional direction. The phenomenon was quantified in Figure 8 for a pyramidal part produced by SPIF with a drawing angle $\psi=60^{\circ}$.

Another conclusion arising from this investigation is that although non-coupled ductile damage models currently utilized in metals cannot apply to the analysis of failure in the hole-flanging produced by PVC because of the reasons that were explained in Section 3.1., the alternative analysis exclusively based on the FFL is reliable and accurate. In fact, the PVC blanks with pre-cut holes of $90 \mathrm{~mm}$ that were able to successfully withstand all the six intermediate forming steps of multistage SPIF, have the strain envelope corresponding to the last forming stage placed below the FFL (Figure 9a). On the contrary, the PVC blanks with pre-cut holes of $80 \mathrm{~mm}$ that failed by fracture during the fifth forming stage (corresponding to $\psi_{5}=85^{\circ}$ ) exhibit a strain envelop inside the grey band area (i.e. inside the uncertainty interval of the FFL) and very close to the FFL (Figure 9b). In connection to this, it is worth noting that the influence of the uncertainty interval of the FFL on the overall performance of the process can be avoided if the sheet polymer parts are designed in order to have strain envelops below the lower bound of the grey band area.

\section{Conclusions}

The proposed methodology to determine the inplane stresses and the accumulated damage at various positions over the surface of conical and pyramidal PVC parts directly from the experimental strain measurements avoids the need to utilize cumbersome inverse procedures based on numerical simulations.
The in-plane stresses are dependent on the strength differential effect of PVC but its influence was found to be rather limited (below 10\%). This result opens the possibility of performing the calculations exclusively based on the tensile stress-strain curve and, thereby, avoids the need to perform stack compression tests.

The critical values of damage associated to standard non-coupled models due to Rice and Tracey and Ayada et al. experience 55 and $53 \%$ variation when the loading conditions change from plane-strain to biaxial stretching. This result prevents their utilization for assessing the formability limits of the SPIF of PVC sheets.

The critical value of damage associated to the noncoupled model due to Cockcroft and Latham experiences a smaller but still important variation (21\%) with the change in loading conditions. This result derives from the fact that iso-damage lines have a slope equal to ' $-1 / 2$ ' (instead of ' -1 ') and, therefore, are closer to the slope ' -0.19 ' of the fracture forming limit line (FFL) of PVC.

However, the overall results indicate that non-coupled damage models currently utilized in sheet metal forming applications should not be applied to characterize formability in the SPIF of PVC. The utilization of the FFL not only proved successful to describe formability in single-stage SPIF but also in multistage SPIF with complex strain paths.

\section{Acknowledgements}

Paulo Martins would like to acknowledge the financial support provided by the German Research Foundation (DFG) under research project SFB/TR 73 during his sabbatical license at the Institute of Forming Technology and Lightweight Construction of the Technical University of Dortmund.

Sammer Alkas Yonan would like to acknowledge the financial support provided by the German Research Foundation (DFG) under research project TE508/20-1.

\section{References}

[1] Glover G., Duncan J. L., Embury J. D.: Failure maps for sheet metal. Metals Technology, 4, 153-159 (1977). DOI: 10.1179/030716977803292006

[2] Franzen V., Kwiatkowski L., Martins P. A. F., Tekkaya A. E.: Single point incremental forming of PVC. Journal of Materials Processing Technology, 209, 462-469 (2008).

DOI: 10.1016/j.jmatprotec.2008.02.013 
[3] Le V. S., Ghiotti A., Lucchetta G: Preliminary studies on single point incremental forming for thermoplastic materials. International Journal of Material Forming, 1, 1179-1182 (2008). DOI: $10.1007 / \mathrm{s} 12289-008-0191-0$

[4] Martins P. A. F., Kwiatkowski L., Franzen V., Tekkaya A. E., Kleiner M.: Single point incremental forming of polymers. CIRP Annals - Manufacturing Technology, 58, 229-232 (2009).

DOI: $10.1016 /$ j.cirp.2009.03.095

[5] Silva M. B., Martinho T. M., Martins P. A. F.: Incremental forming of hole-flanges in polymer sheets. Materials and Manufacturing Processes, 28, 330-335 (2013). DOI: $10.1080 / 10426914.2012 .682488$

[6] Marques T., Silva M. B., Martins P. A. F.: Single point incremental forming of polymers. in 'Mechatronics and manufacturing engineering - Research and development' (Ed.: Davim J. P.) Woodhead Publishing, Oxford, 293-331 (2012).

[7] Silva M. B., Alves L. M., Martins P. A. F.: Single point incremental forming of PVC: Experimental findings and theoretical interpretation. European Journal of Mechanics - A/Solids, 29, 557-566 (2010).

DOI: 10.1016/j.euromechsol.2010.03.008

[8] Alkas Yonan S., Haupt P., Kwiatkowski L., Franzen V., Brosius A., Tekkaya A. E.: A viscoplastic material model based on overstress for the simulation of incremental sheet forming of thermoplastics. AIP Conference Proceedings, 1353, 803-808 (2011). DOI: $10.1063 / 1.3589614$

[9] Alkas Yonan S., Soyarslan C., Haupt P., Kwiatkowski L., Tekkaya A. E.: A simple finite strain non-linear visco-plastic model for thermoplastics and its application to the simulation of incremental cold forming of polyvinylchloride (PVC). International Journal of Mechanical Sciences, 66, 192-201 (2013).

DOI: $10.1016 /$ j.ijmecsci.2012.11.007

[10] Caddell R. M., Raghava R. S., Atkins A. G.: Pressure dependent yield criteria for polymers. Materials Science and Engineering, 13, 113-120 (1974).

DOI: $10.1016 / 0025-5416(74) 90179-7$
[11] Alves L. M., Martins P. A. F.: Cold expansion and reduction of thin-walled PVC tubes using a die. Journal of Materials Processing Technology, 209, 42294236 (2009). DOI: $10.1016 /$ j.jmatprotec.2008.11.015

[12] Spitzig W. A., Richmond O.: Effect of hydrostatic pressure on the deformation behavior of polyethylene and polycarbonate in tension and in compression. Polymer Engineering and Science, 19, 1129-1139 (1979).

DOI: $10.1002 /$ pen.760191602

[13] Lee J. H., Oung J.: Yield functions and flow rules for porous pressure-dependent strain-hardening polymeric materials. Journal Applied Mechanics, 67, 288-297 (2000).

DOI: $10.1115 / 1.1305278$

[14] Cockcroft M. G., Latham D. J.: Ductility and the workability of metals. Journal of the Institute of Metals, 96, 33-39 (1968).

[15] McClintock F. A.: A criterion for ductile fracture by the growth of holes. Journal of Applied Mechanics, 35, 363-371 (1968). DOI: $10.1115 / 1.3601204$

[16] Rice J. R., Tracey D. M.: On the ductile enlargement of voids in triaxial stress fields. Journal of the Mechanics and Physics of Solids, 17, 201-217 (1969). DOI: 10.1016/0022-5096(69)90033-7

[17] Ayada M., Higashino T., Mori K.: Central bursting in extrusion of inhomogeneous materials. Advanced Technology of Plasticity. Proceedings of the $2^{\text {nd }}$ International Conference on Technology of Plasticity, Stuttgart, Germany, 1, 553-558 (1987).

[18] Atkins A. G.: Fracture in forming. Journal of Materials Processing Technology, 56, 609-618 (1996). DOI: 10.1016/0924-0136(95)01875-1

[19] Cristino V. A., Montanari L., Silva M. B., Atkins A. G., Martins P. A. F.: Fracture in single point incremental forming predicted by ductile fracture mechanics. International Journal of Mechanical Sciences, in press (2013). 